Full length article

\title{
Study on the deformations of the lamina cribrosa during glaucoma
}

\author{
Hanjing Tian ${ }^{a}$, Long Li $^{a}$, Fan Song ${ }^{a, b, *}$ \\ ${ }^{a}$ State Key Laboratory of Nonlinear Mechanics (LNM), Institute of Mechanics, Chinese Academy of Sciences, Beijing 100190, China \\ ${ }^{\mathrm{b}}$ School of Engineering Science, University of Chinese Academy of Sciences, Beijing 100049, China
}

\section{A R T I C L E I N F O}

\section{Article history:}

Received 1 November 2016

Received in revised form 14 March 2017

Accepted 16 March 2017

Available online 18 March 2017

\section{Keywords:}

Lamina cribrosa

Glaucoma

Deformation

Optic nerve injury

Reissner's thin plate theory

\begin{abstract}
A B S T R A C T
The lamina cribrosa is the primary site of optic nerve injury during glaucoma, and its deformations induced by elevated intraocular pressure are associated directly with the optic nerve injury and visual field defect. However, the deformations in a living body have been poorly understood yet so far. It is because that integral observation and precise measurement of the deformations in vivo are now almost impossible in the clinical diagnosis and treatment of glaucoma. In the present study, a new mechanical model of the lamina cribrosa is presented by using Reissner's thin plate theory. This model accurately displays the stress and deformation states in the lamina cribrosa under elevated intraocular pressure, in which the shear deformation is not presented by the previous models, however, is demonstrated to play a key role in the optic nerve injury. Further, the deformations of the structures, involving the optic nerve channels and the laminar sheets in the lamina cribrosa, are first investigated in detail. For example, the dislocation of the laminar sheets reaches $18.6 \mu \mathrm{m}$ under the intraocular pressure of $40 \mathrm{mmHg}$, which is large enough to damage the optic nerve axons. The results here confirm some previously proposed clinical speculations on the deformations of the pore shape in the lamina cribrosa under elevated intraocular pressure during glaucoma. Finally, some essentially clinical questions existed during glaucoma, such as the pathological mechanism of the open-angle glaucoma with normal intraocular pressure, are discussed. The present study is beneficial to deeply understanding the optic nerve injury during glaucoma.
\end{abstract}

\section{Statement of Significance}

The lamina cribrosa is the primary site of the optic nerve injury induced by elevated intraocular pressure during glaucoma. Under high intraocular pressure, the optic nerve channel near to the periphery of the lamina cribrosa (Channel A) is deformed to become into a tortuous elliptical horn from a straight cylinder, while the optic nerve channel near to the center of the lamina cribrosa (Channel B) is deformed to become into a straight horn from a straight cylinder. These deformations cause both the axoplasm flow obstacle in the axon fibers and the blocked blood flow in the capillaries which pass through the channels, and trigger the visual field defect during glaucoma.

(c) 2017 Acta Materialia Inc. Published by Elsevier Ltd. All rights reserved.

\section{Introduction}

Glaucoma is the most common cause of irreversible blindness and its pathological mechanism is ascribed substantially to the irreversible deformation of the lamina cribrosa (LC) induced by elevated intraocular pressure (IOP) [1,2]. It is this deformation that results in the damage of the optic nerves passing through the LC and then triggers the visual field defects [3]. Therefore, the deformation states of the LC play a key role in the course of glaucoma

\footnotetext{
* Corresponding author at: State Key Laboratory of Nonlinear Mechanics (LNM), Institute of Mechanics, Chinese Academy of Sciences, Beijing 100190, China.

E-mail address: songf@lnm.imech.ac.cn (F. Song).
}

developing. However, the deformation processes and states of the LC in a living body have been poorly understood yet so far. It is because that integral observation and precise measurement of the deformations in vivo are now almost impossible in the clinical diagnosis and treatment of glaucoma [4]. So, building an appropriate mechanical model of the LC still remains the most efficient way to understand the stress and deformation states of the LC in a living body.

In the previous studies on the mechanical model of the LC, Chi et al. [5] employed a membrane model to investigate the deformation of the LC under elevated IOP. However, because of the reason that the membrane model neglected the thickness and flexural 
resistance of the LC, the results given by the model caused the overestimation on the deformation of the $\mathrm{LC}$.

Considering the thickness and flexural resistance of the LC, Dongqi and Zeqin [6] used Kirchhoff's thin plate theory to first study the deformation of the LC induced by elevated IOP. Because the classical Kirchhoff's thin plate theory inherently includes the postulation that the normal line of the middle plane of plate before the deformation of the plate still remains the normal line of the middle plane after the deformation, i.e. the thin plate theory intrinsically ignores the shear deformation of the plate [7], Dongqi and Zeqin's model is only suitable for roughly obtaining the deformation of the LC with both large radius and small thickness. In fact, in terms of the average dimensions of the LC given by Sigal et al. [8], the deformation given by Dongqi and Zeqin's model was usually much less than the experimental results measured by Levy and Crapps [9]. Even more remarkable is the fact that the model stemming from Kirchhoff's thin plate theory could not, in principle, be employed to study the dislocation between the laminar sheets in the LC, which is proved to play a key role in determining the damage of the optic nerve bundles in the LC.

Edwards and Good [10] used the large deflection plate bending theory to model the deformation of the LC. In terms of the LC with small dimensions, their results are in roughly agreement with the existing experimental results [9]. However, for the LC with average or large dimensions [8], the results given by Edwards and Good's model have considerable errors relative to the experimental data [9]. According to the measurements given by Sigal et al. [8], on the one hand, the thickness of the LC ranges from 0.24 to $0.36 \mathrm{~mm}$, and its average thickness is $h=0.3 \mathrm{~mm}$. On the other hand, Levy and Crapps [9] experimentally obtained the deflection of the LC under IOP $=60 \mathrm{mmHg}$ to be roughly $w=0.03 \mathrm{~mm}$. Therefore, $w / h=0.1$, i.e. the deflection of the LC under high IOP is still less than the thickness of the LC. Based on the theory of plates [7], modeling the deflection of the LC should apply the theory of the plates generating small deflection rather than large deflection. Moreover, the boundary of the model was assumed as a clamped edge without in-plane scleral pretension, which implied that the scleral tension induced by elevated IOP does not affect the stress and deformation in the LC [11].

In addition, there have been many studies using finite element method to calculate the deformation of the LC up to now [11-14]. However, because of the reason that the optic nerve channels and laminar sheets in the LC have both small dimensions and tanglesome structures, employing finite element method hardly obtains the accurate deformation of the structures.

In particular, in order to understand the boundary effects of the mechanical model of the LC, Newson and El-Sheikh [11] detailedly investigated the mechanical models of the LC with six types of boundary conditions by using Kirchhoff's thin circular plate and membrane theories. Their results indicated that a small deflection model of the plate with a clamped edge and considered in-plane pretension due to scleral expansion was the most appropriate to determining the deformation of the LC.

In this paper, based on Reissner's thin plate theory [7], which gives up Kirchhoff's postulation that a normal line of the middle plane of a plate is still a normal line of the middle plane of the plate after deformation, a new mechanical model of the LC is presented and is proved to be in good agreement with the existing experimental data. Employing this model, we firstly obtain the radial, tangential and shear deformations of the LC, and analyze the characteristics of the deformations. In particular, the shear deformation, which is not given by the existing models $[5,6,11]$, is obtained and proved to play a key role in the deformation of the LC and the optic nerve injury in the LC. Secondly, the deformations of the structures including the optic nerve channels and laminar sheets in the LC are first investigated in detail. The results theoret- ically confirm the speculations of Quigley et al. [15] on the deformations on the pores and laminar sheets in the LC induced by elevated IOP in the course of glaucoma developing. Finally, we use the present model to discuss some essential clinical questions existed during glaucoma, involving the pathological mechanism of the open-angle glaucoma with normal intraocular pressure, the intrinsic connection between the ratio of optic cup to disc and the deformations, the damages of the optic nerve bundles induced by the deformations.

\section{Statistical methods}

The experimental data are presented as mean \pm standard deviation. The error bars correspond to the standard deviation. The statistical data were analyzed using the one-way and two-way analyses of variance and examined by paired $t$-test where $p<0.05$ was considered statistically significant. In order to evaluate the goodness of fit of analytically calculated results from different models to the existing experimental data, two statistics: R-square and root mean square error (RMSE) are determined. Statistical analysis was performed using Microsoft Excel software.

\section{Mechanical model}

According to the existing experimental results on the geometrical and mechanical characteristics of the LC $[8,9]$, on the one hand, the thickness of the LC ranges from 0.24 to $0.36 \mathrm{~mm}$, and its average thickness is $h=0.3 \mathrm{~mm}$, while the radius of the LC ranges from 0.76 to $1.14 \mathrm{~mm}$, and its average value is $a=0.95 \mathrm{~mm}$. On average, $h /(2 a)=0.16$ and $h / R_{s}=0.025$, which implies that the LC should be deemed as a thin plate [7], where $R_{s}=12 \mathrm{~mm}$ is the average radius of the eyeball. On the other hand, when IOP is experimentally increased to $60 \mathrm{mmHg}$, the deflection of the LC is measured to be roughly $w=0.03 \mathrm{~mm}$. So, $w / h=0.1$, i.e. the $\mathrm{LC}$ should be considered to be a small deflection plate [7].

So, we reasonably model the LC as a thin elastic flat Reissner's plate undergoing small deflection subjected to a uniformly distributed lateral load, $q=$ IOP - ICP, and a clamped edge with the in-plane pretension, $N$, as shown in Fig. 1 , where ICP is intracranial pressure, which is also the retrolaminar pressure or the optic nerve tissue pressure in previous literatures $[6,10,11]$. The internal forces of Reissner's plate in polar coordinates are expressed by [7],

$M_{r}=-D\left(\frac{d \varphi_{r}}{d r}+v \frac{\varphi_{r}}{r}\right), M_{\theta}=-D\left(\frac{\varphi_{r}}{r}+v \frac{d \varphi_{r}}{d r}\right)$

and

$Q_{r}=C\left(\frac{d w}{d r}-\varphi_{r}\right)$

where $M_{r}$ and $M_{\theta}$ are the radial and tangential moments, respectively; $Q_{r}$ is the shearing force; $w$ is the deflection of the middle plane of the LC, and $\varphi_{r}$ is the rotation angle of the normal line of the middle plane of the plate; $D=E h^{3} /\left[12\left(1-v^{2}\right)\right]$ and $C=5 G h / 6$ are separately the flexural rigidity and the transverse shear stiffness; $E, G$ and $v$ are Young's modulus, the shear modulus and Poisson's ratio of the $\mathrm{LC}$, respectively.

The equilibrium equations of the LC are written by [7]

$\frac{M_{r}-M_{\theta}}{r}+\frac{d M_{r}}{d r}-Q_{r}=0$

and

$Q_{r}=-N_{r} \frac{d w}{d r}-\frac{1}{2} q r$

where $N_{r}$ is the radial tensile force on the cross section of the LC. 
(a)

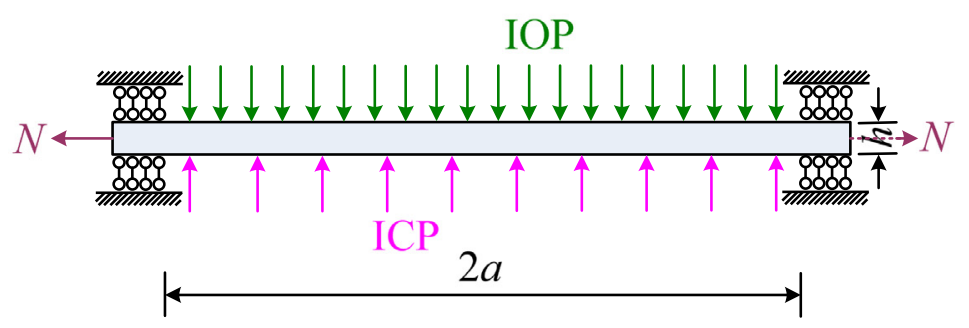

(b)

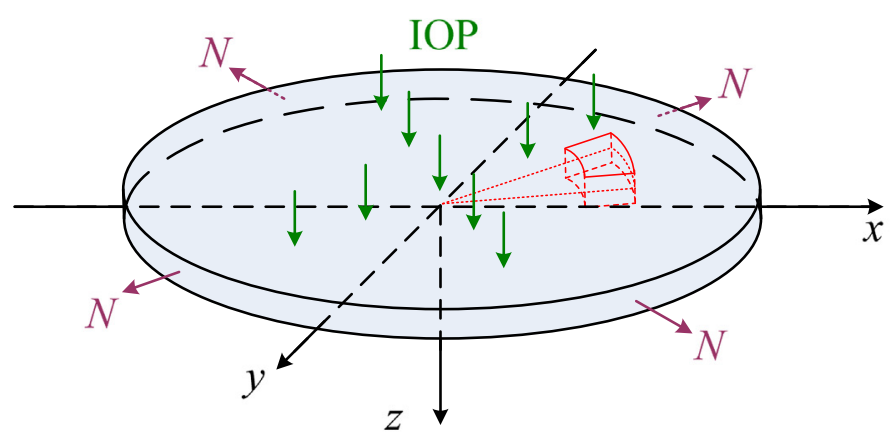

(c)

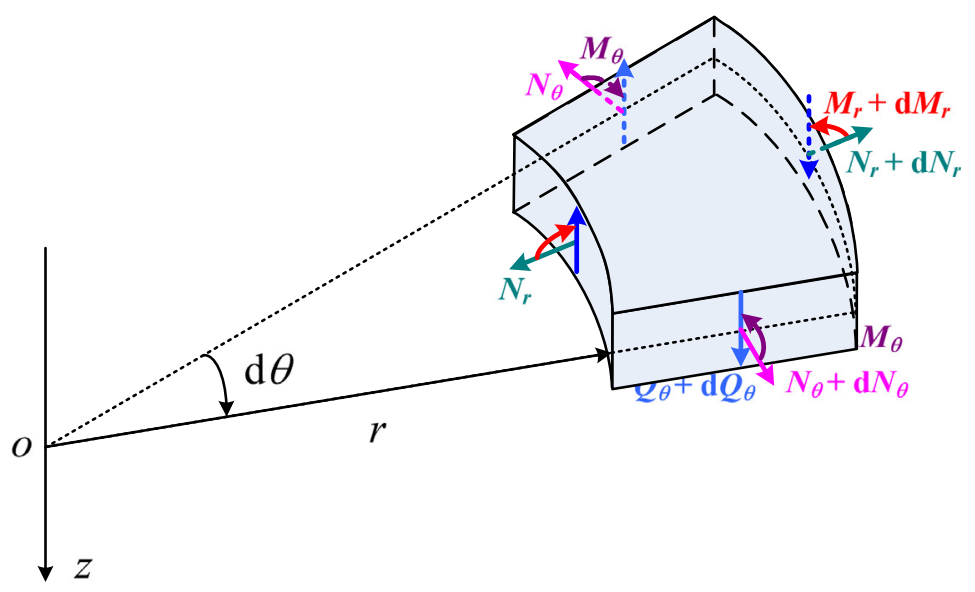

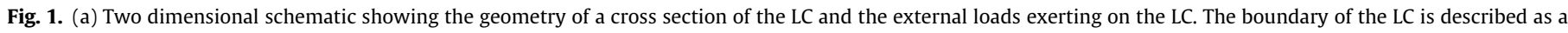

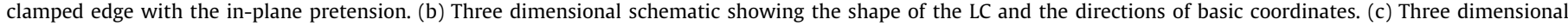
schematic showing the internal forces exerting on an element in the LC.

From Eqs. (1)-(4), we obtain the equation of the rotation angle,

$r^{2} \frac{d^{2} \varphi_{r}}{d r^{2}}+r \frac{d \varphi_{r}}{d r}-\left(\frac{k^{2}}{a^{2}} r^{2}+1\right) \varphi_{r}=\frac{q r^{3}}{2 D\left(1+\frac{N_{r}}{C}\right)}$

where

$k^{2}=\frac{N_{r} a^{2}}{D\left(1+\frac{N_{r}}{C}\right)}$

In Eqs. (5) and (6), generally speaking, $N_{r}$ varies with the coordinates, $r$. However, based on the theory of plates [7], when $(d w /$ $d r)^{2} \ll 1, N_{r}$ has little change under a constant lateral load, $q$. In fact, it is roughly of the order of $10^{-3}$ in the present model. In particular, here the LC is approximated to be an isotropic elastic circu- lar plate under the combined action of a symmetrical lateral load $q$ and a uniform peripheral scleral tension $N$, as shown in Fig. 1. The value of the in-plane pretension $N$ arises from the scleral expansion, and $N=\mathrm{IOP} \cdot R_{S} / 2[6,11]$. Obviously, on the periphery connecting the LC and the sclera, $N_{r}=N$. Therefore, in the present model, we only consider $N_{r}$ to be a constant and equal to $N$, i.e.

$N_{r}=N=\frac{\mathrm{IOP} \cdot R_{S}}{2}$

Thus, Eq. (5) becomes a Bessel equation, and its solution is written by

$\varphi_{r}=C_{1} I_{1}\left(\frac{k}{a} r\right)-\frac{q r}{2 N}$ 
Substituting Eq. (8) into Eqs. (2) and (4) yields the deflection of the $\mathrm{LC}$,

$w=\frac{1}{1+\frac{N}{C}}\left[C_{1} \frac{a}{k} I_{0}\left(\frac{k}{a} r\right)-\frac{q r^{2}}{4 N}-\frac{q r^{2}}{4 C}+C_{2}\right]$

where $I_{0}$ and $I_{1}$ are the modified Bessel functions; $C_{1}$ and $C_{2}$ are integral constants.

The boundary conditions of the LC are efficiently proved both theoretically [11] and experimentally [16,17] to be a clamped edge with the in-plane pretension $N$, as showed in Fig. 1. Using the clamped boundary conditions, i.e. $\varphi_{r}=0$ and $w=0$ at $r=a$, we obtain the integral constants,

$C_{1}=\frac{q a}{2 N I_{1}(k)}$

$C_{2}=-\frac{q a^{2}}{2 N k I_{1}(k)} I_{0}(k)+\frac{q a^{2}}{4 N}+\frac{q a^{2}}{4 C}$

Thus, the rotation angle of the normal line of the middle plane is written by

$\varphi_{r}=\frac{q a}{2 N I_{1}(k)} I_{1}\left(\frac{k}{a} r\right)-\frac{q r}{2 N}$

and the deflection of the LC is expressed by

$w=\frac{q}{1+\frac{N}{C}}\left[\frac{a^{2}-r^{2}}{4 N}+\frac{a^{2}-r^{2}}{4 C}-\frac{a^{2}\left[I_{0}(k)-I_{0}\left(\frac{k}{a} r\right)\right]}{2 N k I_{1}(k)}\right]$

Eq. (13) displays the deflection that the LC is considered as a Reissner's plate. The values of the deflection are associated intimately with the transverse shear stiffness of the plate, $C$. When the transverse stiffness approaches to infinity, i.e. $C \rightarrow \infty$, Eq. (13) implied that any transverse shear deformation does not occur in the LC, and at the same time, Eq. (13) naturally degenerates into the result of Kirchhoff's plate $[6,7,11]$. The results given by the present model are obviously larger than the previous results given by Kirchhoff's plate method [6,11], and the differences between the two results increase nonlinearly with elevated IOP, as shown in Fig. 2a. In the computations here, we use a new relation between IOP and ICP [18], ICP $=10 \operatorname{erf}(0.088$ IOP $)$, which is proved to overcome the induced mathematical singularity in applying the existing relation between IOP and ICP $[6,10,11]$ to compute the mechanical properties of the LC and to be more precise than the existing relation, where $\operatorname{erf}(\xi)=(2 / \sqrt{\pi}) \int_{0}^{\xi} \mathrm{e}^{-\eta^{2}} \mathrm{~d} \eta$ is Gauss error function. In addition, we employ the main mechanical and geometrical parameters of the LC [8] to be $E=0.3 \mathrm{MP}, v=0.49, a=950 \mu \mathrm{m}$ and $h=300 \mu \mathrm{m}$ in the computations of this study.

The deflection given by Eq. (13) is compared with the existing experimental data [9] and models [6,10], as shown in Fig. 2b. We calculated two statistics: R-square and RMSE to evaluate the goodness of fit of different models, as shown in Table 1. From this Table, $\mathrm{R}$-square is closer to 1 and RMSE is closer to 0 in our present model. Therefore, the present model is obviously in better agreement with the experimental results than the previous models.

\section{Results}

\subsection{Deformation analysis}

Based on Eq. (13), the radial normal strain is written by [7]

$\varepsilon_{r}=-z\left\{\frac{q k}{2 N I_{1}(k)}\left[I_{0}\left(\frac{k}{a} r\right)-\frac{a}{k r} I_{1}\left(\frac{k}{a} r\right)\right]-\frac{q}{2 N}\right\}$

On the one hand, this radial normal strain linearly varies on a cross section of the LC ( $r=$ const.): it is equal to zero on the middle plane of the $\operatorname{LC}(z=0)$, and it reaches its tensile and compressive a)

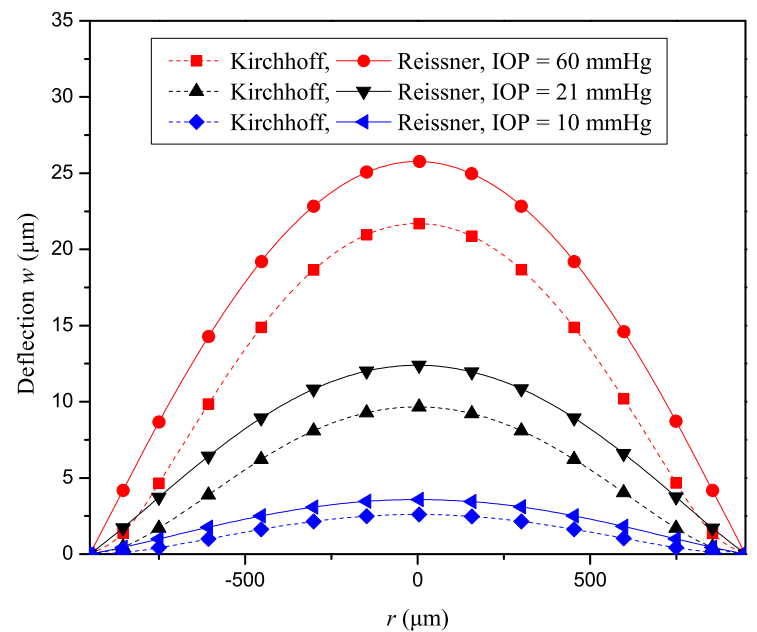

b)

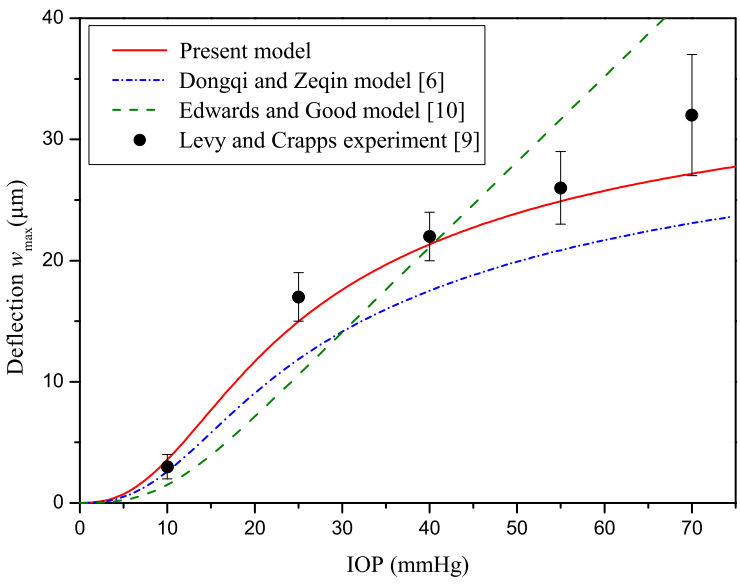

Fig. 2. Calculation graphs (a) showing the deflections of the LC caused by different IOP, which displays that the deflections of LC given by Reissner's thin plate model is greater than that given by Kirchhoff's model; and (b) showing the deflections of the center of the LC with IOP variation, which derive from the different mechanical models and experiment.

Table 1

R-square and root mean squared errors (RMSE) of different models with respect to the experiments of Levy and Crapps [9].

\begin{tabular}{llll}
\hline Statistics / Model & Present & $\begin{array}{l}\text { Dongqi and } \\
\text { Zeqin [6] }\end{array}$ & $\begin{array}{l}\text { Edwards and } \\
\text { Good [10] }\end{array}$ \\
\hline R-square & 0.939855 & 0.683257 & 0.627365 \\
RMSE $(\mu \mathrm{m})$ & 2.407904 & 5.525758 & 5.993496 \\
\hline
\end{tabular}

maximum values on the cross section at the anterior and posterior surfaces of the LC $(z= \pm h / 2)$, as shown in Fig. 3a. On the other hand, the strain is the symmetry with respect to $r$ - coordinate axis. The anterior part of the central region of the $\mathrm{LC}(r<600 \mu \mathrm{m}, z<0)$ is subject to a compressive strain, while the posterior part of the central regions $(r<600 \mu \mathrm{m}, z>0)$ is subject to a tensile strain. On the contrary, the anterior part of the limbic region of the LC $(r>600 \mu \mathrm{m}, z<0)$ is subject to a tensile strain, while the posterior part of the limbic regions $(r>600 \mu \mathrm{m}, z>0)$ is subject to a compressive strain, as shown in Fig. 3b. Here, the limbic region of the LC is defined as the region of $r>600 \mu \mathrm{m}$. This indicates that there is a transition point between the central and limbic regions of the LC, at which the radial deformation of the LC does not occur, i.e. 
a)

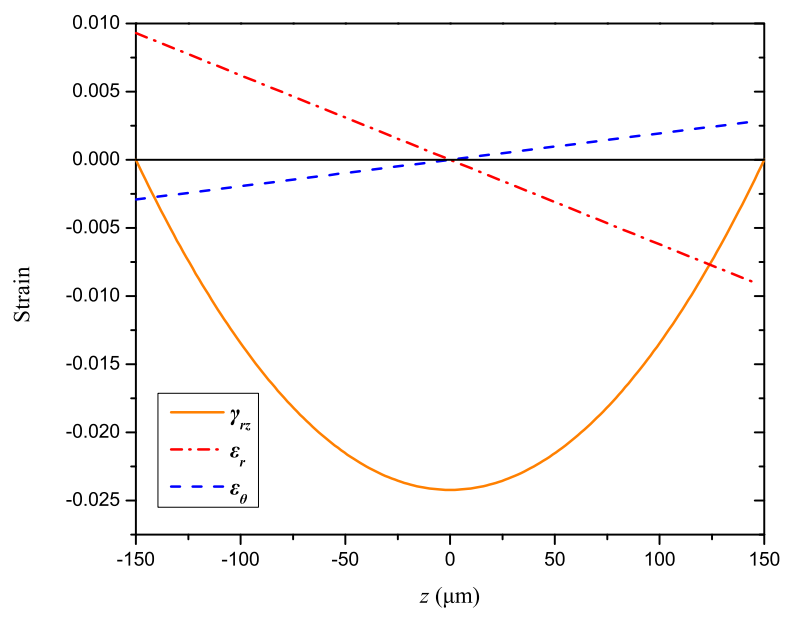

c)

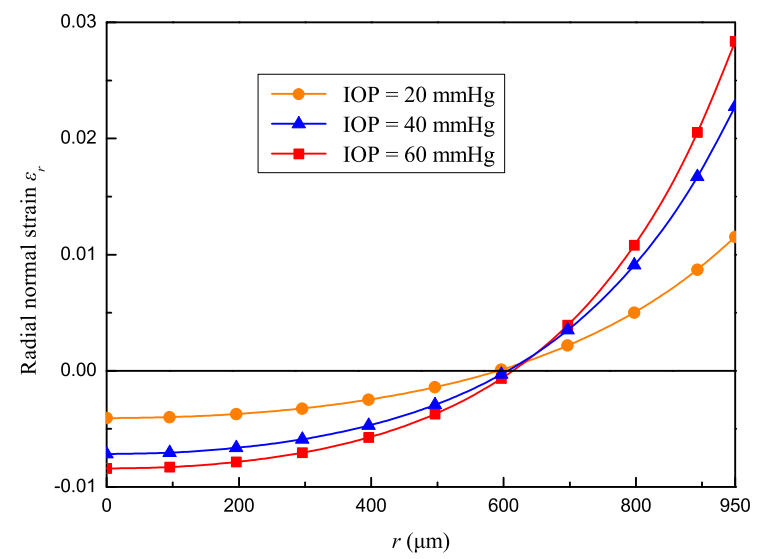

b)

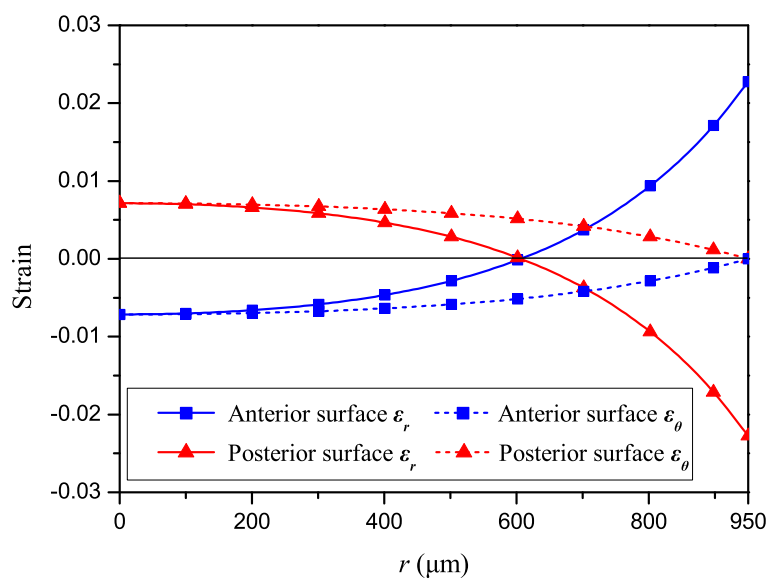

d)

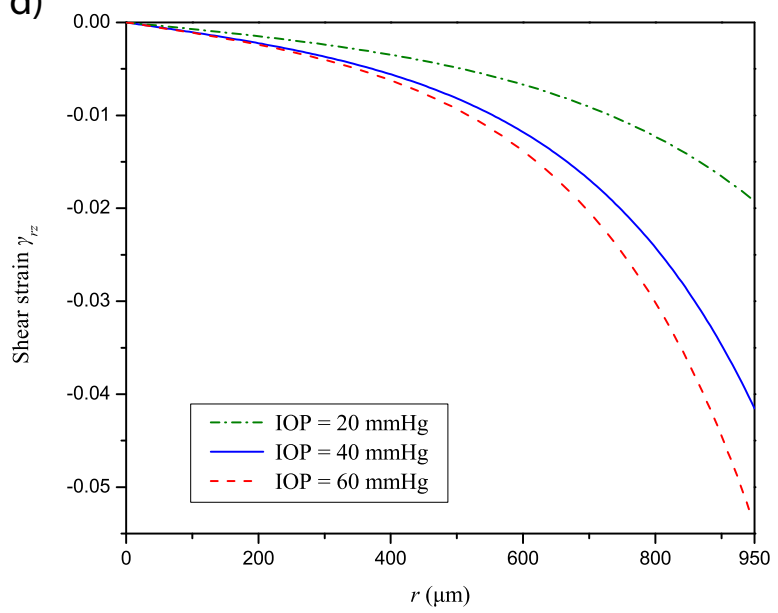

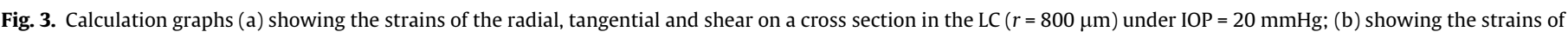

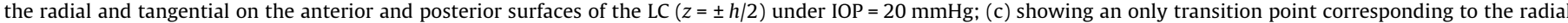

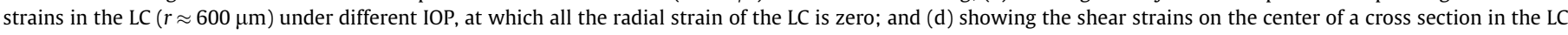
$(r=800 \mu \mathrm{m}, z=0)$ under different IOPs.

$\varepsilon_{r}=0$ at $r \approx 600 \mu \mathrm{m}$. Further, we prove that the position of the transition point is only relative to the radius and thickness of the LC, and is scarcely relevant to the changes of IOP, as shown in Fig. 3c. The meaning of the point will be discussed in following section. In addition, we readily compute that the maximum values of the tensile and compressive strains in whole the LC occur at the anterior and posterior edges of the LC $(r=a, z= \pm h / 2)$, respectively, as shown in Fig. 3b.

The tangential normal strain of the $\mathrm{LC}$ is written by

$\varepsilon_{\theta}=-z\left[\frac{q a}{2 r N I_{1}(k)} I_{1}\left(\frac{k}{a} r\right)-\frac{q}{2 N}\right]$

This tangential normal strain also linearly varies on a cross section of the LC ( $r=$ const.). It is also equal to zero on the middle plane of the $\operatorname{LC}(z=0)$ and reaches the tensile and compressive maximum values on the cross section at the anterior and posterior surfaces of the LC $(z= \pm h / 2)$, as shown in Fig. 3a. In the direction of $r$ - coordinate axis, the strain monotonously decreases from the center to the edge of the LC. The whole anterior surface of the LC is subject to a compressive strain, while the whole posterior surface is subject to a tensile strain, i.e. at the center of the plate $(r=0)$ the strain has the maximum and at the rim of the LC $(r=a)$ the strain is equal to zero, as shown in Fig. 3b. Obviously, the maximum values of the tensile and compressive strains in whole the LC occur at the anterior and posterior centers of the LC $(r=0, z= \pm h / 2)$, respectively. Note that at the center of the LC $(r=0)$ the values of the tangential and radial normal strains are the same. by

The shear strain on an arbitrary cross section of the LC is written

$\gamma_{r z}=-\frac{3 q a}{G h^{3}\left(1+\frac{N}{C}\right)}\left(\frac{h^{2}}{4}-z^{2}\right) \frac{I_{1}\left(\frac{k}{a} r\right)}{I_{1}(k)}$

Obviously, this strain displays a parabolic distribution with respect to $z$ on a cross section of the LC ( $r=$ const.). At the posterior and anterior surfaces of the plate $(z= \pm h / 2)$, its values are all equal to zero, while its maximum occurs on the middle plane of the plate $(z=0)$, as shown in Fig. 3a. In addition, the shear strain monotonously decreases from the center to the rim of the LC. At the center of the plate $(r=0)$ the strain is equal to zero, while at the rim of the plate $(r=a)$ the strain reaches the maximum, as shown in Fig. $3 \mathrm{~d}$. 


\subsection{Deformations of pores and laminar sheets}

We further investigate the deformations of the pores and laminar sheets in the LC. The LC is a collagen-based meshwork structure [19], which approximately consists of 10 successive sheets [2]. The thickness of the LC ranges from 0.24 to $0.36 \mathrm{~mm}$, the average thickness is $h \approx 0.3 \mathrm{~mm}[3,8]$. So, the average thickness of a lamina sheet is about $30 \mu \mathrm{m}$. The radius of the $\mathrm{LC}$ ranges from 0.76 to $1.14 \mathrm{~mm}$, and the average radius is $a \approx 0.95 \mathrm{~mm}$. In the LC there roughly exist 200-400 channels (or pores), through which the nerve fiber bundles pass entering into the brain. The diameters of the channels range from 10 to greater than $100 \mu \mathrm{m}$, and the average diameter is roughly $l_{0}=50 \mu \mathrm{m}$ [20].

Here two optic nerve channels near separately to the center and rim of the LC are chosen to study their deformations induced by elevated IOP. The centers of the two channels are located at $r=900$ (Channel A) and $100 \mu \mathrm{m}$ (Channel B) in the LC, respectively. And they are assumed to be straight and to have the circular cross section with the same diameter $\left(l_{0}=50 \mu \mathrm{m}\right)$ before deformation, as shown in Fig. 4a. On the one hand, when the deflection of the LC occurs under IOP $=40 \mathrm{mmHg}$, based on Eqs. (14) and (15), the entrance pore of Channel A on the anterior surface of the LC is in the tensile deformation region of the LC, while the exit pore of the channel on the posterior surface of the LC is in the compressive deformation region of the $\mathrm{LC}$, as shown in Fig. $3 \mathrm{~b}$. We readily obtain that at the entrance and exit pores of Channel $A$, the radial and tangential strains of the pore center are separately $\varepsilon_{r} \approx \pm 0.03$ and $\varepsilon_{\theta} \approx \pm 0.002$. So, the diameter changes of the entrance and exit pores are calculated to be roughly $l_{0} \varepsilon_{r} \approx \pm 1.5 \mu \mathrm{m}$ and $l_{0} \varepsilon_{\theta} \approx \pm 0.10 \mu \mathrm{m}$, respectively. This is an indication that both the circular pores located on the anterior and posterior surfaces of the LC change into ellipses, as shown in Fig. $4 \mathrm{~b}$, which is in very agreement with the speculations given by Quigley et al. [15] on the deformations of the pore shape in the LC under elevated IOP. In detail, on the anterior surface of the LC after the deformation, the major axis of the entrance ellipse pore is $l_{0}+l_{0} \varepsilon_{r} \approx 51.5 \mu \mathrm{m}$ and the minor axis of the pore is $l_{0}+l_{0} \varepsilon_{\theta} \approx 50.10 \mu \mathrm{m}$, while on the posterior surface, the major axis of the exit ellipse pore is $l_{0}-l_{0} \varepsilon_{\theta} \approx 49.90 \mu \mathrm{m}$ and the minor axis of the pore is $l_{0}-l_{0} \varepsilon_{r} \approx 48.5 \mu \mathrm{m}$. Note that after the deformation, the direction of the major axis of the entrance ellipse pore is in $r$ - coordinate axis, while the direction of the major axis of the exit ellipse pore is in $\theta$ - coordinate axis, which are vertical to one another, as shown in Fig. 4b.

On the other hand, based on Eq. (16), the shear strain does not make the deformation of both the entrance and exit pores on the anterior and posterior surfaces of the LC, however, it results in the deformation of the interior of Channel $\mathrm{A}$ and the dislocation of the laminar sheets in the LC. We compute the shear strain at the central location of Channel A to be roughly $\gamma_{r z}(r=900 \mu \mathrm{m}$, $z=0) \approx 0.062$, which is the maximum value of the shear strain of the channel under IOP $=40 \mathrm{mmHg}$. So, the maximum displacement occurs at the center of Channel $\mathrm{A}$ in the direction of $r$ - coordinate axis, the value of which is $\gamma_{r z} h \approx 18.6 \mu \mathrm{m}$. And in the direction of $z$ - coordinate axis, the displacement induced by the shear strain decreases in parabolic route from the middle plate to the surfaces of the LC, as shown in Fig. 4b. Therefore, the resultant of the three strains makes Channel A become into a tortuous horn from an initially straight cylinder, which is also in agreement with the speculations of Quigley et al. [15] on the deformations of the pores and the laminar sheets in the LC induced by elevated IOP.

The deformation of Channel B differs from that of Channel A. Because the position of Channel B is near to the center of the LC, the deformation caused by the shear strain is negligible according to Eq. (16). So, we only investigate the contributions of the radial and tangential normal strains to the deformation of Channel $\mathrm{B}$. Based on Eqs. (14) and (15), the entrance pore of Channel B on the anterior surface of the LC is in the compressive deformation region of the $\mathrm{LC}$, while the exit pore of the tunnel on the posterior surface of the LC is in the tensile deformation region of the plate, as shown in Fig. 3b. Using Eqs. (14) and (15), we readily calculate that

\section{(a) Before deformation}

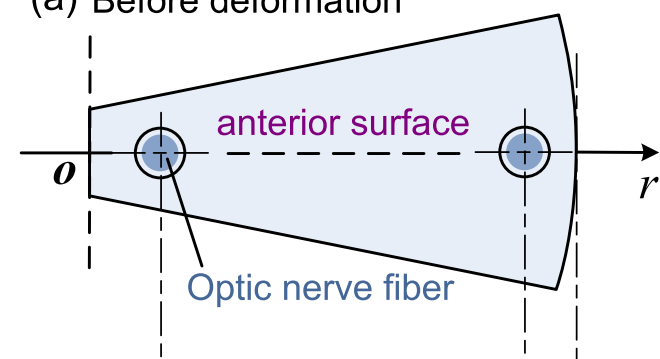

Channel B

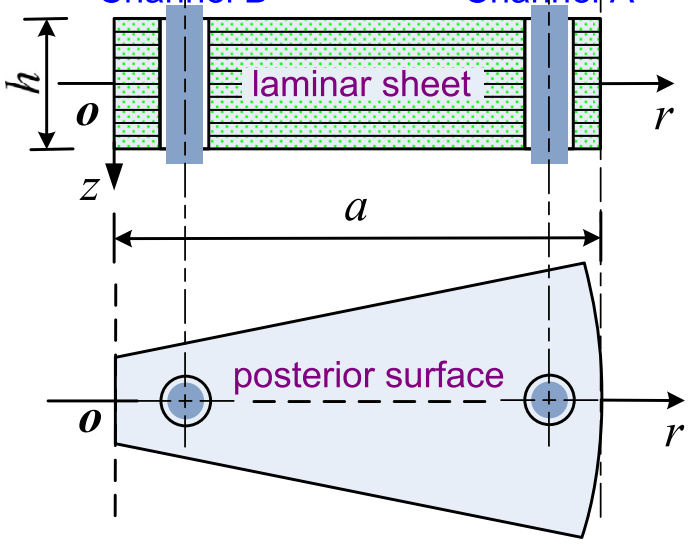

\section{(b) After deformation}

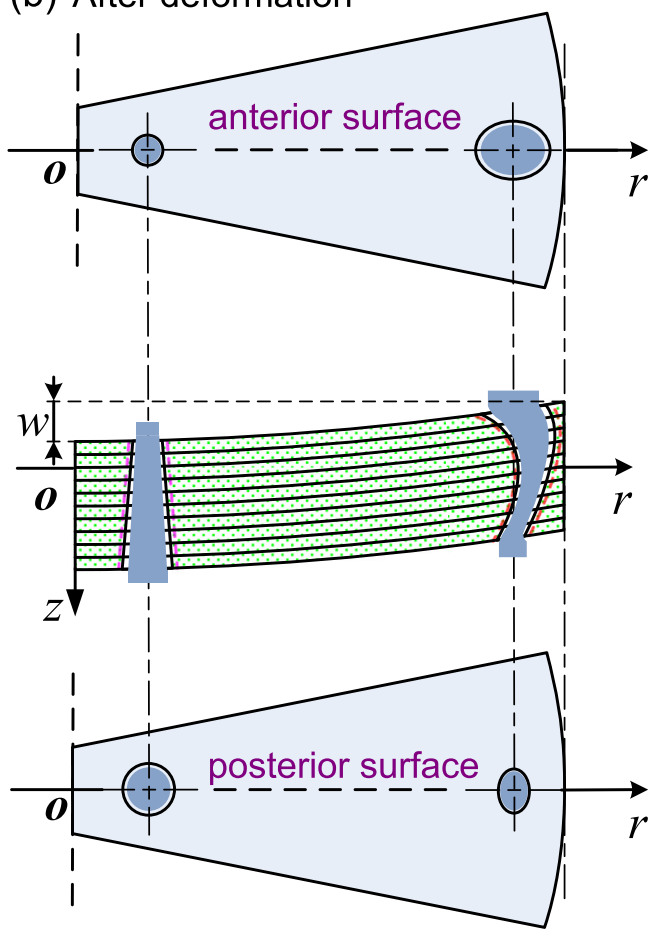

Fig. 4. Schematics (a) and (b) showing the states of the optic nerve channels and laminar sheets in the LC before and after deformation, respectively. 
at the exit and entrance of the channel, the radial and tangential strains at the two pore centers are the same and roughly equal to $\varepsilon_{r} \approx \varepsilon_{\theta} \approx \pm 0.01$. Therefore, both the shapes of the exit and entrance pores are not changed by the two strains and still remain circular. However, the diameter of the exit pore on the posterior surface of the LC increases to $l_{0}+l_{0} \varepsilon_{r}=50.5 \mu \mathrm{m}$, while the diameter of the entrance pore on the anterior surface of the LC decreases to $l_{0}-l_{0} \varepsilon_{r}=49.5 \mu \mathrm{m}$. The shape of Channel B becomes into a straight horn from an initially straight cylinder, as shown in Fig. 4b, which is also in agreement with the speculations of Quigley et al. [15] on the deformations of the pores and the laminar sheets in the LC induced by elevated IOP.

Further, comparing the deformations of the Channels A and B, we find that the pores near to the rim of the LC produce larger deformation than the pores near to the center of the LC under elevated IOP. This result means that the optic nerve bundles passing through the pores near to the rim are more easily damaged than the bundles passing through the pores near to the center of the LC, which is in agreement with the experiments [2,20,21]. Obviously, with the increase of IOP, the deformations of both the optic nerve channels and laminar sheets in the LC induced by the strains will increase nonlinearly according to Eqs. (14)-(16).

\section{Discussion}

The present model is based on Reissner's rather than Kirchhoff's thin plate theory, i.e. all results given by the model radically include the contributions of the transverse shear effects in the LC under elevated IOP. So, the model must be more accurately to provide the real mechanical states of the LC than the existing models based on Kirchhoff's thin plate theory. For example, the present model can obtain the shear deformation in the LC, Eq. (16), which is proved to play an important role in investigating the deformation of the channels and laminar sheets in the LC. However, in the existing models of the LC $[5,6,11]$, there is no transverse shear deformation according to Kirchhoff's postulation of thin plate theory [7].

The deformations of the LC, Eqs. (14)-(16), are theoretically controlled by the two forces vertical to one another, $q=$ IOP - ICP and $N=\left(\right.$ IOP $\left.\cdot R_{s}\right) / 2$, as shown in Fig. 1 . The increased lateral load, $q$, tends to compress the LC deflection towards posterior, while the increased scleral tension, $N$, pulls the LC expansion towards rim and simultaneously impedes the deflection of the LC. However, when IOP $\geq 20 \mathrm{mmHg}$, ICP is confirmed to be roughly a constant, ICP $=10 \mathrm{mmHg}[2,6,10,11]$. Therefore, in fact, IOP becomes into only force that causes the deformation of the LC under high IOP. It is proved that IOP plays a key role in the course of glaucoma developing. In particular, employing the present model, we find that under the condition that the pressure difference, $q$, remains unchanged, the deflection produced by decreasing ICP is greater than the deflection produced by increasing IOP in the LC, as shown in Fig. 5. This is probably an explanation for the open-angle glaucoma with normal IOP [22].

Based on Eq. (13), the deflection of the LC increases with the decrease of the thickness under the same IOP: the less the thickness, the larger its deflection, while the deflection increase with the increase of the radius: the larger the radius, the larger the deflection. Correspondingly, the deformation of the $\mathrm{LC}$ is in keeping with the changes of the deflection with respect to the thickness and radius according to Eqs. (14)-(16). The experimental results of previously studies indicated that the black subjects, on average, have a larger $L C$ radius than white subjects $[5,23]$. It implies that the deflection of the LC in blacks will be greater at any given level of IOP according to the above analysis. The results here may partly explain racial differences in glaucoma incidence, which shows that

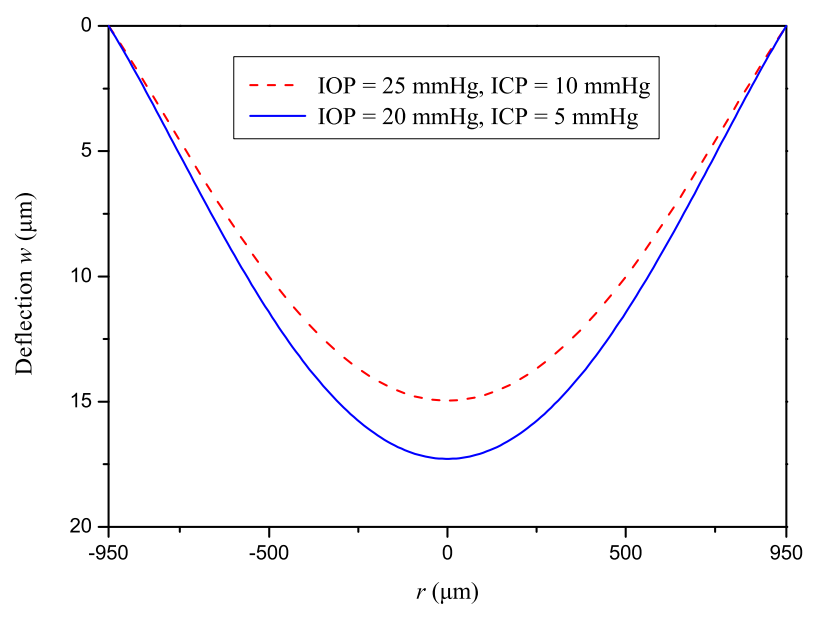

Fig. 5. Calculation graph showing the deflections of the LC caused by the same pressure difference ( $q=\mathrm{IOP}-\mathrm{ICP})$ and different pressures.

the blacks are more susceptible than the whites to the damages of the LC during glaucoma [5].

The ratio of the diameter of optic cup to that of optic disc, C/D, is one of the principle physiological characteristics in the diagnosis of glaucoma and now clinically becomes into a warning structural parameter in judging glaucoma to occur. C/D of the normal human eye ranges from 0.3 to 0.6 . Once C/D is greater than a critical value, about 0.6 , the glaucoma will usually be confirmed in clinical practice [24]. In the view of eye structures, the diameter of optic disc is equal roughly to that of the LC, i.e. $2 a \approx \mathrm{D}$. And the diameter of optic cup, in clinical practice, is the width of a depression measured near to the optic nerve head, which is associated necessarily with the deformation of the LC. Based on the analysis of Eq. (14) above, there is a transition point of the radial deformation of the $\mathrm{LC}, r_{\mathrm{t}} \approx 600 \mu \mathrm{m}$, at which the radial deformation is equal to zero, $\varepsilon_{r}=0$. And it is proved that the position of the transition point is only relative to the geometry of the LC and is scarcely relevant to the changes of IOP. In the view of mathematics, the transition point stands for a deflection point of the Eq. (13), i.e. $d^{2} w / d r^{2}=0$, at which the deformation of the LC has the largest gradient in the radial direction. Therefore, the transition point is as very similarly significant as the position of the optic cup in the clinic. In particular, $r_{\mathrm{t}} / a \approx 600 / 950 \approx 0.63$ is in agreement with the critical value of $C / D$, which might inherently display the meaning of the critical valve of $C / D$.

As the primary site of retinal ganglion cell axon injury during glaucoma $[2,25,26]$, the LC stands for the intervening link between elevated IOP and the optic nerve injury. Therefore, the present model, serving as a formalistic expression of the optic nerve injury during glaucoma, makes a probability to deeply understand this disease. As analyzed above, on the one hand, the elevated IOP results in the area expansion of the entrance pores of the optic nerve channels near to the rim of the LC. This induces the increase of the inflow volumes in both the optic nerve bundles and blood capillaries that pass through the channels. However, the IOP also results in the area contraction of the exit pores of the channels. This necessarily makes the squeezing each other of the optic nerve bundles and blood capillaries, and causes the decrease of the outflow volumes in both the optic nerve bundles and blood capillaries. On the other hand, the elevated IOP simultaneously causes the deflections of the channels, as shown in Fig. 4b. The maximum value of the deflection reaches $18.6 \mu \mathrm{m}$, which is as the same order of magnitude as the thickness of a laminar sheet. The dislocation is much greater than the diameters of the optic nerve axon fibers and blood capillaries, which range from 0.65 to $1.10 \mu \mathrm{m}$ [27]. In view of 
fluid mechanics, it is enough to cause the axoplasm flow obstacle in the axon fibers and the blocked blood flow in the capillaries $[28,29]$. In addition, the deformation of the channels near to the rim of the LC is much greater than that near to the center of the LC according to the present model. Therefore, the damage of the optic nerves near to the rim occurs necessarily earlier and heavier than that near to the center, which is in agreement with the existing clinical tests [30].

According to the theory of plates [7], we can use Eq. (13) to obtain readily the stress states of the LC, including the stresses of the perimeter zones of the optic nerve channels and the interfaces between arbitrary two laminar sheets in the LC. This must be very beneficial to further understanding the deformation of the LC and the damage of the optic nerve in the LC.

As a limitation of our model, the LC is assumed to undergo elastic deformation when it is subjected to external loads. In practice, the mechanical properties of biological materials depend strongly on the interactions of their individual components. It has been experimentally indicated that the LC is likely to be viscoelastic [31,32]. Albon et al. [32] showed that the stiffness of the LC increases with the level of applied pressure by volume and strain measurements. To further study the stress and deformation states of the LC induced by elevated IOP, the viscoelastic properties of the LC can be taken into consideration and the establishment of viscoelastic constitutive relation for the LC under elevated IOP will be of crucial importance.

Generally speaking, the elevated IOP is considered the primary factor responsible for the glaucomatous optic neuropathy involving the death of retinal ganglion cells and the loss of their axons, and the control of IOP remains the mainstay of treatment for glaucoma. However, it should be important to note that there are many other pathophysiological mechanisms involved in the development of retinal ganglion cell death and glaucomatous optic neuropathy. Recent studies have revealed that the vascular dysregulation also plays a critical role in these progresses. Additionally, the apoptotic cell death in glaucoma has been attributed to various molecular mechanisms including glutamate excitotoxicity, increased nitric oxide synthase-2 expression, tumor necrosis factor-alpha upregulation, increased matrix metalloproteinase expression and oxidative stress. It is the complex interplay of these multiple factors that leads to the apoptosis of retinal ganglion cell and glaucomatous optic neuropathy $[25,33]$.

\section{Conclusions}

Based on Reissner's thin plate theory, a mechanical model of the LC is presented and is proved to be in good agreement with the existing experiments. Employing this model, we firstly obtain the radial, tangential and shear deformations of the LC, and then analyze the characteristics of the deformations. Secondly, the deformations of the optic nerve channels and laminar sheets in the LC are investigated in detail, which theoretically confirms the speculations of Quigley et al. Finally, we use this model to mainly discuss a possible pathological mechanism of the open-angle glaucoma with normal IOP, the relations between the ratio of optic cup to disc in the clinical practice and the deformation of the LC, and the damages of the optic nerve bundles induced by the deformation of the LC. The present study is beneficial to understanding deeply the pathological mechanism of glaucoma, in particular, the damage of the optic nerves in the LC.

\section{Conflict of interest}

There are no conflicts of interest to report in this work.

\section{Acknowledgments}

The work was supported by the programs in the National Natural Science Foundation of China (Grants 11232013 and 11472285), the Strategic Priority Research Program of the Chinese Academy of Sciences (Grant XDB22040102) and the National Key Research and Development Program of China - China (Grant 2016YFA0501601).

\section{References}

[1] H.A. Quigley, A.T. Broman, The number of people with glaucoma worldwide in 2010 and 2020, Br. J. Ophthalmol. 90 (2006) 262-267.

[2] H.A. Quigley, Pathophysiology of optic nerve in glaucoma, in: J.A. McAllister, R. P. Wilson (Eds.), Glaucoma, Butterworths, London, 1986, pp. 30-53.

[3] M.J.A. Girard, N.G. Strouthidis, A. Desjardins, J.M. Mari, C.R. Ethier, In vivo optic nerve head biomechanics: performance testing of a three-dimensional tracking algorithm, J. R. Soc. Interface 10 (2013) 1-13.

[4] J.C. Downs, Optic nerve head biomechanics in aging and disease, Exp. Eye Res. 133 (2015) 19-29.

[5] T. Chi, R. Ritch, D. Stickler, Racial differences in optic nerve head parameters, Arch. Ophthalmol. 107 (1989) 836-839.

[6] H. Dongqi, R. Zeqin, A biomathematical model for pressure-dependent lamina cribrosa behavior, J. Biomech. 32 (1999) 579-584.

[7] S. Timoshenko, S. Woinowsky-Krieger, Theory of Plates and Shells, McGrawHill Book Company Inc., New York, 1959.

[8] I.A. Sigal, J.G. Flanagan, C.R. Ethier, Factors influencing optic nerve head biomechanics, Invest. Ophthalmol. Visual Sci. 46 (2005) 4189-4199.

[9] N.S. Levy, E.E. Crapps, Displacement of optic nerve head in response to shortterm intraocular pressure elevation in human eyes, Arch. Ophthalmol. 102 (1984) 782-786.

[10] M.E. Edwards, T.A. Good, Use of a mathematical model to estimate stress and strain during elevated pressure induced lamina cribrosa deformation, Curr. Eye Res. 23 (2001) 215-225.

[11] T. Newson, A. El-Sheikh, Mathematical modeling of the biomechanics of the lamina cribrosa under elevated intraocular pressures, J. Biomech. Eng. - T. ASME 128 (2006) 496-504.

[12] A.J. Bellezza, R.T. Hart, C.F. Burgoyne, The optic nerve head as a biomechanical structure: initial finite element modeling, Invest. Ophthalmol. Visual Sci. 41 (2000) 2991-3000.

[13] I.A. Sigal, J.G. Flanagan, I. Tertinegg, C.R. Ethier, Finite element modeling of optic nerve head biomechanics, Invest. Ophthalmol. Visual Sci. 45 (2004) $4378-4387$.

[14] I.A. Sigal, C.R. Ethier, Biomechanics of the optic nerve head, Exp. Eye Res. 88 (2009) 799-807.

[15] H.A. Quigley, R.W. Flower, E.M. Addicks, D.S. McLeod, The mechanism of opticnerve damage in experimental acute intraocular-pressure elevation, Invest. Ophthalmol. Visual Sci. 19 (1980) 505-517.

[16] S.L.Y. Woo, A.S. Kobayashi, W.A. Schlegel, C. Lawrence, Nonlinear material properties of intact cornea and sclera, Exp. Eye Res. 14 (1972) 29-39.

[17] I.A. Sigal, J.G. Flanagan, I. Tertinegg, C.R. Ethier, Modeling individual-specific human optic nerve head biomechanics. Part II: influence of material properties, Biomech. Model. Mechanobiol. 8 (2009) 99-109.

[18] H.J. Tian, R.Q. Du, F. Song, A modified relation between the intraocular and intracranial pressures, Theor. Appl. Mech. Lett. 6 (2016) 148-150.

[19] Z. Nadler, B. Wang, G. Wollstein, J.E. Nevins, H. Ishikawa, R. Bilonick, L. Kagemann, I.A. Sigal, R.D. Ferguson, A. Patel, D.X. Hammer, J.S. Schuman, Repeatability of in vivo 3D lamina cribrosa microarchitecture using adaptive optics spectral domain optical coherence tomography, Biomed. Opt. Express 5 (2014) 1114-1123.

[20] H.A. Quigley, E.M. Addicks, Regional differences in the structure of the lamina cribrosa and their relation to glaucomatous optic-nerve damage, Arch. Ophthalmol. 99 (1981) 137-143.

[21] H.A. Quigley, R.M. Hohman, E.M. Addicks, R.W. Massof, W.R. Green, Morphologic changes in the lamina cribrosa correlated with neural loss in open-angle glaucoma, Am. J. Ophthalmol. 95 (1983) 673-691.

[22] R.J. Ren, J.B. Jonas, G.G. Tian, Y. Zhen, K. Ma, S.N. Li, H.T. Wang, B. Li, X.J. Zhang, N.L. Wang, Cerebrospinal fluid pressure in glaucoma a prospective study, Ophthalmology 17 (2010) 259-266.

[23] L. Dandona, H.A. Quigley, A.E. Brown, C. Enger, Quantitative regional structure of the normal human lamina cribrosa. a racial comparison, Arch. Ophthalmol. 108 (1990) 393-398.

[24] J.G. Crowston, C.R. Hopley, P.R. Healey, A. Lee, P. Mitchell, The effect of optic disc diameter on vertical cup to disc ratio percentiles in a population based cohort: the blue mountains eye study, Br. J. Ophthalmol. 88 (2004) 766-770.

[25] H.A. Quigley, E.M. Addicks, W.R. Green, A.E. Maumenee, Optic nerve damage in human glaucoma. II. The site of injury and susceptibility to damage, Arch. Ophthalmol. 99 (1981) 635-649.

[26] Y.H. Kwon, J.H. Fingert, M.H. Kuehn, W.L. Alward, Primary open-angle glaucoma, N. Engl. J. Med. 360 (2009) 1113-1124.

[27] B.M. Johnson, M. Miao, A.A. Sadun, Age-related decline of human optic-nerve axon populations, Age 10 (1987) 5-9. 
[28] G. Guidoboni, A. Harris, L. Carichino, Y. Arieli, B.A. Siesky, Effect of intraocular pressure on the hemodynamics of the central retinal artery: a mathematical model, Math. Biosci. Eng. 11 (2014) 523-546.

[29] G. Guidoboni, A. Harris, S. Cassani, J. Arciero, B. Siesky, A. Amireskandari, L. Tobe, P. Egan, I. Januleviciene, J. Park, Intraocular pressure, blood pressure, and retinal blood flow autoregulation: a mathematical model to clarify their relationship and clinical relevance, Invest. Ophthalmol. Visual Sci. 55 (2014) 4105-4118.

[30] D.F. Garway-Heath, D. Poinoosawmy, F.W. Fitzke, R.A. Hitchings, Mapping the visual field to the optic disc in normal tension glaucoma eyes, Ophthalmology 107 (2000) 1809-1815.
31] A.J. Bellezza, C.J. Rintalan, H.W. Thompson, J.C. Downs, R.T. Hart, C.F. Burgoyne, Deformation of the lamina cribrosa and anterior scleral canal wall in early experimental glaucoma, Invest. Ophthalmol. Vis. Sci. 44 (2003) 623-637.

[32] J. Albon, P.P. Purslow, W.S.S. Karwatowski, D.L. Easty, Age related compliance of the lamina cribrosa in human eyes, Br. J. Ophthalmol. 84 (2000) 318-323.

[33] R. Agarwal, S.K. Gupta, P. Agarwal, R. Saxena, S.S. Agrawal, Current concepts in the pathophysiology of glaucoma, Indian J. Ophthalmol. 57 (2009) 257-266. 